\title{
ON SIMULATION-BASED WARGAMING: COMPARISON OF TWO DIFFERENT METHODOLOGICAL APPROACHES
}

\author{
Marko A. HOFMANN and Tobias LEHMANN
}

\begin{abstract}
Wargaming has been neglected within the German Forces for almost fifty years. Only within the last two years, two so-called "Leitfaden" (codes of best practice) have been developed. The first one, elaborated by members of the General Staff Academy, is intended for operational wargaming and fully rests on the traditional analytical comparison of different Courses of Action (CoA). Own and enemy CoAs are played against each other on the foundation of a game between two staff groups. Within this paradigm, simulations are used as evaluation tools. The reasoning is based on the premise that simulation systems are valid representations of reality. A successful simulation run is considered a corroboration of a particular course of action. The second "Leitfaden," developed at the University of the Federal Armed Forces, is intended for so-called "tactical wargaming." The two central concepts of this approach are intuition-based decision-making and assumptionbased planning. The core rationale of this method is that all military planning and decision making is liable to what is called the "fog of war." The irresolvable unpredictability of future developments in all military operations is considered an insurmountable obstacle to all analytical approaches. This approach rests on the claim that pattern recognition and mental simulation of these patterns are the most valuable cognitive functions of expert decision makers. Within this paradigm, simulations are used to support creativity, enhance imagination and trigger self-critical thinking. Simulation systems of complex systems are not necessarily regarded to be valid. This article discusses the pros and cons of the two approaches ("Leitfaden") from both theoretical and practical perspective.
\end{abstract}

Keywords: Wargaming, Operational Wargaming, Tactical Wargaming, Simulation, Intuition-based Decision-making, Assumption-based Planning

\section{On Wargaming - Introduction}

"Wargaming is a flexible instrument designed to develop, compare and improve Courses of Action (COA)." ${ }^{1}$ This is the definition given for wargaming at operational level by the General Staff College of the Federal Armed Forces. 
The origin of institutionalized wargaming dates back to emerging Prussia and its General Staff in the $19^{\text {th }}$ Century and can be described as means to interactively play the development of military (and later on non-military as well) operations. ${ }^{2}$ Both, Clausewitz and Moltke the Elder saw the potential of wargaming in a staff being better prepared for an operation within a common context, the usage of its members' creativity and intensified contingency planning by critically questioning assumptions and validating planned COAs. The wargaming process is not bound to a certain level of command, but its implementation strongly depends on this, since each level of command has typical constraints concerning available time, resources and experts in different operation-specific fields. Typically, tactical, operational and strategic levels are differentiated. In this article, the focus is on tactical level but in order to give a more comprehensive picture on wargaming, the differences with operational level are introduced as well.

Tactical level (here brigade level) sets up tight time boundaries for the decisionmaking processes, such as 30 minutes to a few hours maximum for a planning horizon of no more than 48 hours. Within this time period, decision-making, order production and distribution of orders as well as briefing of subordinate commanders have to take place, which additionally reduces the leeway for wargaming to about a fourth or less of the total available time.

It should be clear that the support (may it be computers, maps, papers, order templates, blackboards, etc.) has to meet the requirements of the fairly low time available for initialization and use. Furthermore, the necessary level of expertise for use of the support system has to be (permanently) available. As it is known from decision theory and described by leading researchers such as Jeffrey, Chernoff, Moses, Keeney and Raiffa, ${ }^{3}$ the rational analysis of a certain situation is time consuming due to the uniqueness of warlike situations ${ }^{4}$ and, therefore, the continuous necessity for creation of new models or customization and revalidation of the already developed ones emerges as a crucial obstacle for the IT-support in general.

\section{Operational Wargaming}

The main steps of the high level process of operational wargaming are illustrated in Figure 1, following the representation provided by Birnstiel. ${ }^{5}$ The cyclical, iterative nature of the process could easily be seen. This process focuses on the cyclic gaming of COAs according to the Action-Reaction-Counteraction pattern.

After this, in order to prepare commander's decision, a decision matrix is set up, which rates each COA in different ways. To this end, several methods are proposed, such as the Advantage/ Disadvantage Table and the four-dimensional SWOT (Strength, Weakness, Opportunities, Threats) Matrix, as representatives of non-for- 


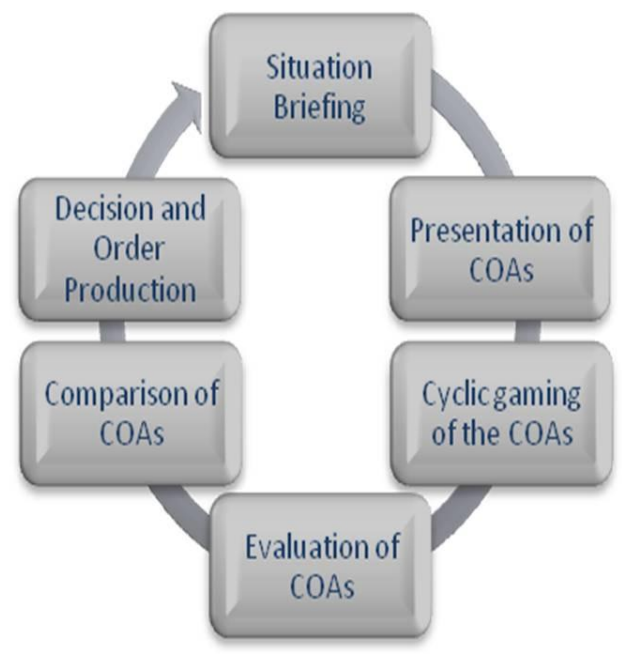

Figure 1: Main Steps of Operational Wargaming.

mal and therefore non-numerical methods. More formal methods are the use of colorbased (ordinal order) or numerical (cardinal order) matrices as shown in Figure 2. While the ordinal approach (presented on the left hand side part of the Figure) allows discrete ratings, which are not comparable in a way that a "++" is double beneficial as a "+" or four times better than a " 0 ", it is still very well suited for getting an overview of COAs, which is technically similar to the Advantage/ Disadvantage Matrix. But due to its ordinal ratings, a comparison is equal to a multi-objective evaluation with non-formalizeable utility functions as described in the work of Keeney and Raiffa, Hadi, and Zimmermann. ${ }^{6}$ This apparent drawback could be compensated using purely numerical matrices, as shown on the right hand side of Figure 2.

However, the use of the word "could" here has to be emphasized. Practically, this is possible in a very limited scope of applications, where hard facts are compared with each other only. In real-world situations, the weighting of different factors and the following totting up of these can lead to the dangerous impression that $\operatorname{COA}(A)$ is better than $\operatorname{COA}(B)$ by 0.5 points and is, therefore, always preferable.

\section{Tactical Wargaming}

At tactical level, there is no even sufficient time for gaming through all COAs as a method for their validation. Therefore, it seems, and three-field exercises have underpinned this hypothesis, that questioning of assumptions made along the planning process or at certain control-points reveals various hidden potholes or even wrong parameter(s) and, therefore, lead to qualitatively better COAs. The authors are aware of 


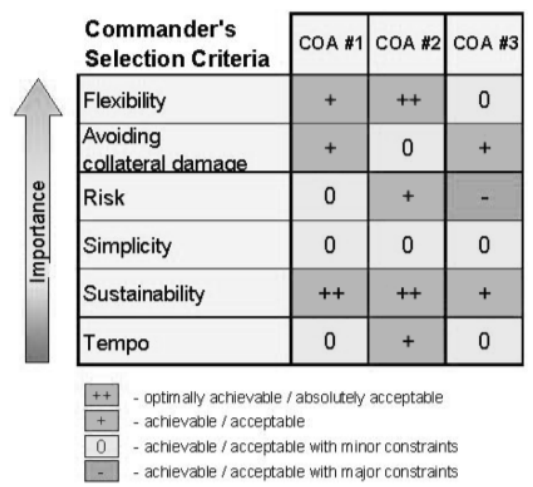

\begin{tabular}{|c|c|c|c|c|c|c|c|}
\hline \multirow[b]{2}{*}{$\begin{array}{l}\text { Commander's } \\
\text { Selection Criteria }\end{array}$} & \multicolumn{3}{|c|}{ COA \#1 } & \multicolumn{2}{|c|}{ COA \#2 } & \multicolumn{2}{|c|}{ COA \#3 } \\
\hline & 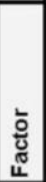 & 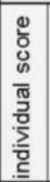 & ङ & 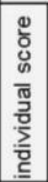 & 퓸 & 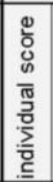 & త్ర \\
\hline \begin{tabular}{|l} 
Avoiding \\
collateral damage
\end{tabular} & 3 & 3 & 9 & 2 & 6 & 3 & 9 \\
\hline Risk & 3 & 2 & 6 & 3 & 9 & 3 & 9 \\
\hline Tempo & 1 & 2 & 2 & 3 & 3 & 2 & 2 \\
\hline Flexibility & 4 & 3 & 12 & 4 & 16 & 2 & 8 \\
\hline Simplicity & 2 & 2 & 4 & 2 & 4 & 1 & 2 \\
\hline Sustainability & 2 & 4 & 8 & 4 & 8 & 3 & 6 \\
\hline & & & 41 & & 46 & & 36 \\
\hline
\end{tabular}

Figure 2: Quantified Evaluation Methods for COAs.

the difficulties faced when rating decisions in unique situation environments, but, in general, we assume that an incorrect assumption is less helpful than a correct one. Even though a decreased number of COAs is used (it is not a must to set up three COAs), the results of this approach exceed the results from cognitive sciences, ${ }^{7}$ where experts tend to come up with one single decision that meets all necessary requirements. Yet, it is not meant to abolish the rational analysis, but more to find a practical way of using the available time more efficiently. In addition, the value of a good answer/ solution found too late is always less than that of a useful answer given in time. In order to give to the process a defined structure in the sense of a framework or a guideline, the following steps (as presented in Figure 3) have been set up. Compared to the operational guideline, this process is presented in a different granularity, but its 0020description is eventually almost identical. Since this process is the focus of this article, its description is given with more details.

The process begins with a visualization of the current situation (Step \#1), which is considered to be of high importance in order to create a common understanding between all members of the staff. Due to the fact that this step is one of the core activities within a brigade staff and is usually well supported by information technologies, there is no much to be added. Step \#2 then separates the current situation from future developments in a way that it requires the own systems to be able to manipulate the representation of the situation without further consequences for the staff work. It adds a mission layer on top of the situation layer and in such a way ensures that everybody 


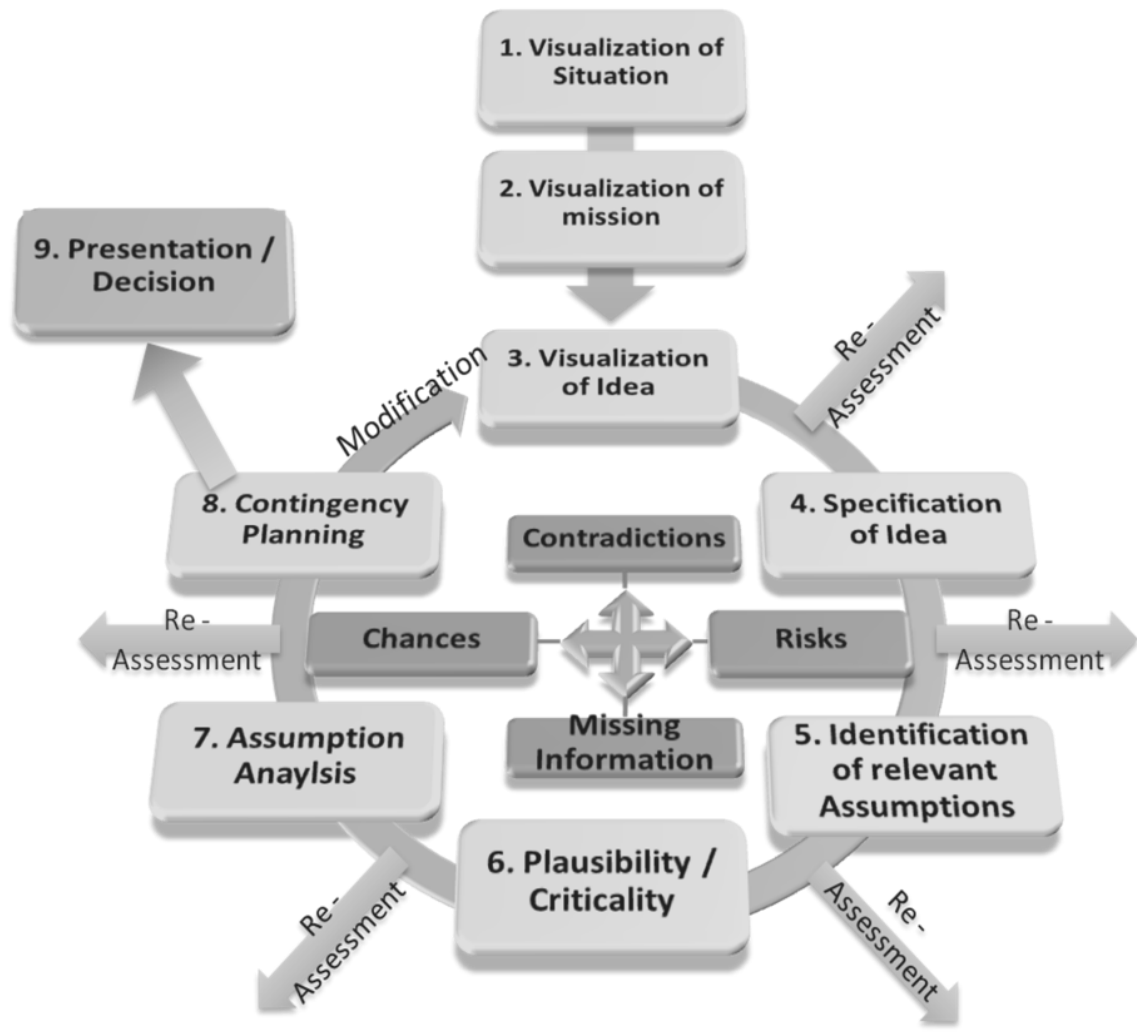

Figure 3: Tactical Wargaming Process.

would talk about the same issues. The third step is first in the creativity-cycle that encompasses Step \#3 to Step \#8, which are all in "the battle" between missing information and overflow of contradictious information, as well as between Chances and Risks for own personnel and materiel, eventually, until the success of the operation. Here, an idea bridges the difference between current situation and defined mission and their objectives. It has to be pointed out that this action has no defined starting point; technically, this initial idea could be of any nature and it is more than a starting point for discussion - it is a well evaluated COA.

Step \#4 then specifies the idea at hand by disassembling it into its core elements using the lowest reasonable (depending on the context) granularity. After this, the idea's elements are subject to analysis for relevant assumptions. The point here is to find a good balance between a search in depth (e.g., recursive questioning of assumptions) and search in width (finding out the largest number of independent assumptions). Usually, due to time limits, it seems better to start searching for a large number of in- 

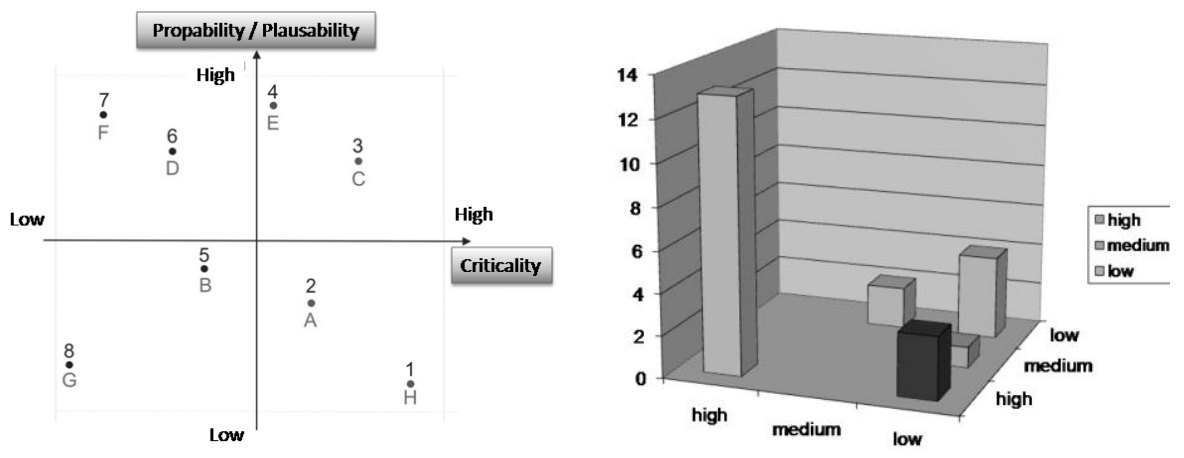

Figure 4: Classification of Assumptions.

dependent assumptions and proceeding then with them to Step\#6. The goal of Step \#6 is to classify the assumptions according to their criticality and plausibility. An example of this procedure is given in Figure 4 (refer to the left-hand side). All used values are not quantified and usually not justified by hard facts or even numerical data. However, this method quickly filters out these assumptions that are highly implausible and, at the same time, due to their criticality - a threat to the operation itself. Step \#7 aims then to find what will happen if an assumption would not hold and here the study is limited to the "dangerous" assumptions defined in Step \#6. As shown in Figure 4, here COTS software such as Microsoft Excel or similar Apple and Open Office products can be of great help to cluster and visualize the assumptions. Taking these results into consideration, Step \#8 addresses contingency plans according to the alternatives defined in Step \#7.

If the contingency plans are free of inconsistencies to a required level, then these plans are made explicit (if time suffices) or it is announced that oral communication is possible only; after that follows presentation of these plans, as well as eventually the order production. If this is not the case, for example a contingency plan reveals a critical threat that has been neglected up to this point, then the commander or the chief of staff can choose to run through the cycle again with certain and limited modifications or to repeat the planning process completely from scratch. This exit option for a complete restart is not limited to be taken after Step \#8 but is possible throughout the whole process, since critical insights will be gained anytime during the planning and refinement phase.

\section{Integration of Simulation into the Wargaming Approaches}

The two presented wargaming methods can - technically-be conducted with very simple equipment: a map, a spreadsheet and some kind of representation for military entities are sufficient. However, a computer-based representation of terrain, tactical 
objects, coordination lines and a video projector enable a much faster and more efficient visualization, as well as a computer-based treelike structuring of the wargaming results significantly simplifies data management. Hence, we strongly recommend such computer-assisted solutions.

It has to be mentioned here that due to time constraints at tactical and operational level, the efficient support by means of simulation systems diverges significantly in nature. Since the operational level has a planning phase of about 48 hours, a more quantitative analysis is possible. Hence, mathematical models can be customized, filled with data and calculated. Or, in case of simulation, intensive interactive runs can be conducted in order not to rely on computer agents but on human experience and reasoning power, too. Results can be described formally and can be integrated into other models as well. However, at tactical level, where only a planning phase of about a couple of hours at maximum is available, the decision support could only be of more qualitative nature. Directions, trends or general statements can be generated, but not a detailed view on complex subjects could be elaborated. ${ }^{8}$

Whereas the general support by information technologies is unquestionable and quite similar for both approaches, the integration of computer simulations into them differs significantly. In operational wargaming, simulations are used to evaluate the consequences of options (COAs) on the basis of statistical parameters like expected value or variance generated by stochastic simulation runs. This approach is, however, limited to data-rich technical systems. It is hardly practical for social systems, where the uncertainty in input data and cause-effect relationships is ubiquitous.

In tactical wargaming, simulations are employed to find hitherto (within the current planning) unexpected events that might occur. It is possible to use both closed and interactive simulation runs. The former have the advantage to allow a large number of simulation runs to be performed and substantial introduction of randomness in modeling bad luck and chance. A major advantage of tactical wargaming is that the validity of the simulation model being applied is not as much important as for operational wargaming, since the simulation is only used as a qualitative thought trigger and not as quantitative evaluation method.

The initial experience gained by the authors during three computer-assisted command post exercises are very encouraging for the use of simulations within tactical wargaming and rather pessimistic about the application of simulations in operational wargaming: There is a high acceptance among the German staff officers for the first and much mistrust concerning the second approach. 


\section{Summary and Conclusion}

This article has considered wargaming at tactical and operational level with its different parameters and constraints. As a basis for discussion, means of decision support for COA evaluation have been presented and the benefits and drawbacks of a strongly formal (numerical decision matrix) evaluation have been discussed. The authors are skeptical that an objective evaluation of COAs is generally possible, since it leaves too much space for subjective interpretation and influence. In addition, the number of not directly measurable parameters (for reasons of missing information or not quantifiable parameters) is too high. Still, the authors see a great potential for decision support in formal models such as simulation, which can be used in both, quantitative and qualitative, decision support as well as at the two wargaming levels. This can be mainly associated with domains of quantifiable problems with generally a little number of social elements. But there is a strong necessity to be aware of its limitations as well as to keep in mind the need for interpretation of elaborated results by model specialists. The integration of simulation systems into wargaming as a part of military staff planning has been tested within a brigade exercise "Golden Shield" in 2006 and 2007 with very important results. It is planned to proceed with these field exercises in order to elaborate wargaming-specific features as add-ons for simulation systems as well as to cooperate intensively with the German Army Warfighting Centre in Wildflecken and Dresden.

\section{Notes:}

1 M. Birnstiel, et al. Wargaming - Guide to Preparation and Execution, B.d. Verteidigung (Bundeswehr Command and Staff College, 2006).

2 Rupert Smith, The Utility of Force: The Art of War in the Modern World, Volume 2 (London: Penguin Books Ltd., September 2005). 
3 Richard C. Jeffrey, The Logic of Decision (New York: McGraw-Hill, 1965); Herman Chernoff and Lincoln E. Moses, Elementary Decision Theory (New York, London, Sydney: John Wiley \& Sons, 1970); Ralph L. Keeney and Howard Raiffa, Decisions with Multiple Objectives: Preferences and Value Tradeoffs (New York, London, Sydney: John Wiley \& Sons, 1978).

4 Carl von Clausewitz, Vom Kriege (Reinbek: Rowohlt Tb., 1978).

5 Birnstiel, Wargaming - Guide to Preparation and Execution.

6 Keeney and Raiffa, Decisions with Multiple Objectives; Fathi A. Hadi, Entscheidungskriterien und Nutzenfunktionen (Frankfurt am Main: Haag \& Herchen Verlag $\mathrm{GmbH}$, 1979); Hans-Jürgen Zimmermann, "Die Formulierung und Lösung schlechtstrukturierter Entscheidungsprobleme," in Grundlagen des Operations Research, ed. Tomas Gal (Berlin, Heidelberg, New York: Springer Verlag, 1987), 340-368.

7 Gary A. Klein, Sources of Power: How People Make Decisions (Cambridge, MA: The MIT Press, 1998); Dominic J. Caraccilo and John L. Pothin, "Coup d'oeil: The Commander's Intuition in Clausewitzian Terms," Air \& Space Power Journal (16 February 2000): 21-28; Phillipp E. Ross, "Wie Genies denken," Spektrum der Wissenschaft 36, no. 1 (2007): 36-43.

8 Jon Doyle and Richmond H. Thomason, "Background to Qualitative Decision Theory," AI Magazine 20, no. 2 (1999): 55-68.

MARKO HOFMANN - Information about the author is available on page 27 of this volume.

TOBIAS LEHMANN - Information about the author is available on page 63 of this volume. 